\title{
Pioglitazone versus metformin in Egyptian non diabetic patients with Non Alcholic Fatty Liver Disease: A randomized controlled trial.
}

\author{
Sahar K. Hgazy a, Mamdouh A. Gabr ${ }^{\text {b }}$, Nahla E. El-Ashmawy ${ }^{\text {c}}$, Maha A. \\ Younis ${ }^{\text {a* }}$ \\ a Clinical Pharmacy Department, Faculty of Pharmacy, Tanta University, Egypt \\ b Internal Medicine Department, Faculty of Medicine, Tanta University, Egypt \\ c Biochemistry Department, Faculty of Pharmacy, Tanta University, Egypt \\ E-mail: mahayounis2013@yahoo.com \\ Corresponding author*
}

\begin{abstract}
NAFLD is estimated to be the most common cause of chronic liver disease and there are increasing rates of NASH-related liver transplantation. This study evaluates the effect of two different insulin sensitizers, pioglitazone and metformin, on liver fat content, lipolysis and insulin sensitivity in Egyptian patients with non alcoholic fatty liver disease. Forty Egyptian patients with fatty liver were enrolled in a six-month prospective study. The patients were recruited from outpatient clinics of Department of Internal Medicine, Tanta University Hospitals with ultrasonographic diagnosis of fatty liver. The patients were randomly divided into two groups; Group I (pioglitazone group) was treated by $45 \mathrm{mg}$ pioglitazone/day and Group II (metformin group) was treated by metformin $20 \mathrm{mg} / \mathrm{kg} / \mathrm{day}$. A third group of healthy subjects served as control group. The study indicated that both antihyperglycemic drugs decreased homeostatic model assessment-insulin resistance $(\mathrm{p}<0.01)$, plasma free fatty acids $(\mathrm{p}<0.01)$ and liver fat content $(\mathrm{p}<0.01)$ associated with improvement of hyperechogenicity in liver ultrasound in both the pioglitazone group $(\mathrm{p}<0.001)$ and the metformin group $(p=0.47)$. Both treatments also improved serum levels of alanine aminotransferase $(p<0.01)$, aspartate aminotransferase $(\mathrm{p}<0.01)$, high density lipoprotein-cholesterol $(\mathrm{p}<0.01)$ and adiponectin $(\mathrm{p}<0.01)$. The insulin sensitizers pioglitazone and metformin exhibited a beneficial role in management of NAFLD. Meanwhile, pioglitazone was superior to metformin in improving liver stestosis and could provide a therapeutic option to guard against disease complications.
\end{abstract}

Key words:-Egyptian; Fatty liver; insulin resistance; pioglitazone; metformin.

\section{INTRODUCTION}

Non-alcoholic fatty liver disease (NAFLD) occurs among all ages and ethnicities and is recognized to occur in 20\%-30\% of the general population [1]. NAFLD is defined as the accumulation of excessive fat in the liver without a history of excessive drinking of alcohol and any secondary cause [2]. Primary NAFLD is related to insulin resistance and strictly associated with metabolic risk factors especially obesity, diabetes and dyslipidemia [3]. NAFLD can progress slowly from simple steatosis that presents without inflammation and hepatocellular damage to non-alcoholic steatohepatitis (NASH) that demonstrates inflammation and hepatocellular damage and subsequently leads to hepatic fibrosis. NAFLD may be progressive resulting in cirrhosis and hepatocellular carcinoma [4]. Accumulation of hepatic fat is closely linked to insulin resistance, which increases lipolysis of peripheral adipose tissue resulting in increased free fatty acids influx to the liver. Moreover, insulin resistance encourages de novo triglyceride synthesis within the liver, decreased triglyceride export through very-low density lipoprotein (VLDL) and inhibits oxidation of fatty acid thereby, promoting triglyceride accumulation [5]. So, improving insulin sensitivity is one of the targets in the treatment of NAFLD.

At present, insulin sensitizing drugs (like pioglitazone and metformin) have come to interest as a source to decrease insulin resistance in NAFLD patients. Pioglitazone, a thiazolidinedione derivative, is a peroxisome proliferator-activated receptor $\gamma$ (PPAR- $\gamma$ ) agonist that improves insulin sensitivity and glucose and lipid metabolism in type II diabetes [6]. Treatment with thiazolidinediones results in consistent decrease in Free Fatty Acids (FFAs) [7], thereby decreasing FFA delivery to the liver. They also increase adiponectin levels which may help to increase lipid oxidation of fatty acids in the liver [6]. Metformin is a member of the biguanide class of drugs. It appears to improve insulin sensitivity by reducing the fat content of liver and muscle through activation of adenine monophosphate (AMP)-dependent protein kinase that results in increased mitochondrial FFA oxidation and decreased FFA and VLDL synthesis [8]. Metformin also improves sensitivity to insulin by 
reducing hepatic gluconeogenesis, reducing lipolysis in adipose tissue, increasing peripheral glucose uptake by liver, skeletal muscle and adipose tissue [9].

The present study was conducted to examine and compare the effect of pioglitazone and metformin on liver fat content and lipid profile in NAFLD patients. We aimed also to correlate their effects to the insulin sensitivity and adiponectin level.

\section{PATIENTS}

Forty Egyptian patients with evidence of NAFLD were included in the study. They were 29 males and 11 females, their ages ranged from 34 to 61 years, their BMI ranged from 26.7 to $42 \mathrm{~kg} / \mathrm{m}^{2}$. The patients were recruited from Internal Medicine Department, Tanta University Hospitals, Tanta. Exclusion criteria included alcohol use (more than $20 \mathrm{~g}$ per day for men and $10 \mathrm{~g}$ per day for women), diabetes mellitus, positive results for tests of autoimmune hepatitis and virus markers (HBS antigen, HCV antibody), heart disease (ischemic or congestive), renal disease (serum creatinine concentration of $>1.5 \mathrm{mg} / \mathrm{dl}$ ), using fibrate or statins medications, previous treatment (with thiazolidinediones, biguanides, or insulin), and pregnant women. The demographic characteristics of the patients were shown in table 1.

The patients were randomly divided into two equal groups: pioglitazone group received Actos® (Takeda Pharmaceuticals, Japan) (45mg/day) and metformin group received Glucoghage ${ }^{\circledR}$ (Merck, Germany) $(20 \mathrm{mg} / \mathrm{kg} /$ day). Twenty healthy control subjects were included in the study and served as the control group. All participants were asked to sign an informed consent form prior to inclusion in the study. The study was approved by the Ethical Committee of Tanta University.

\section{METHODS}

All patients underwent a complete clinical examination by liver ultrasound and laboratory tests at baseline and six-months post treatment. Blood samples were obtained after a 10-12 hours fasting period. Fasting blood glucose was measured immediately, then blood was centrifuged and serum was separated, divided into portions, and stored at $-80{ }^{\circ} \mathrm{C}$ until assayed. Another portion of blood was added to tubes containing EDTA and then plasma was separated after centrifugation. All plasma samples were stored at $-80^{\circ} \mathrm{C}$ until analysis of FFA.

\section{Biochemical assays}

Fasting blood glucose (FBG) levels were assayed using glucoseoxidase method (Spinreact, Spain) [10]. Alanine aminotransferase (ALT) and aspartate aminotransferase (AST) were measured by enzymaticcolorimetric method (Biodiagnostic, Egypt) [11]. Adiponectin [12], fasting insulin [13] and free fatty acids levels were determined using ELISA (Enzyme-Linked Immunosorbent Assay) with commercial kits of adiponectin (Assaypro, USA), insulin (Drg-diagnostics, Germany), and free fatty acids (Cusabio Biotech, China), respectively.

For estimation of lipid profile, total cholesterol [14], triglycerides [15] and high density lipoprotein cholesterol (HDL-C) [16] were measured by enzymatic colorimetric method using commercial kits (BioMed, Germany). Low-density lipoprotein cholesterol (LDL-C) was calculated using the Friedewald formula [17] where LDL-C $=[$ TC - HDL-C $-($ TGs/5)] provided that TGs level is less than $400 \mathrm{mg} / \mathrm{dl}$ [17]. Insulin resistance (IR) was calculated using homeostasis model assessment-IR (HOMA-IR = fasting insulin concentration $(\mu \mathrm{IU} / \mathrm{ml}) \mathrm{x}$ fasting glucose concentration $(\mathrm{mg} / \mathrm{dl}) / 405)[18]$.

\section{Liver Ultrasound}

Liver ultrasound was performed at baseline and 6 months post-treatment and read by a blinded, specialized radiologist. The existence of fat in liver parenchyma scatters the beam of ultrasound more than a non-fatty liver. Therefore, increased echogenicity was considered matched with fatty liver. The diagnosis of liver steatosis is based on (1) hyperechogenicity of the liver relative to adjacent right kidney or spleen, (2) unclear show of intra-hepatic lacuna structures, (3) round and blunt border of the liver with mild to moderate enlargement, (4) unclear show of envelop of right liver lobe and diaphragm [19]. Severity of fatty liver was quantitated according to Roldan-Valadez, et al. [20] using a scoring system $(0=$ no fatty liver, $1=$ mild fatty liver, $2=$ moderate fatty liver and 3 =severe fatty liver). Mild steatosis was characterized by a mild increase in liver echogenicity. Moderate steatosis appeared as increased liver echogenicity that made hepatic and portal vein walls not obvious. Severe steatosis was characterized by posterior attenuation of the deep liver parenchyma [20].

\section{Liver Fat Content Calculation}

Liver biopsy is the gold standard for the diagnosis and staging of NAFLD [21], but performing liver biopsy for the determination of NAFLD has some limitations. Many patients do not agree to make liver biopsy due to its cost and its invasive nature. Liver fat content was calculated as follows: "Liver fat content $(\%)=10$ ($0.805+0.282 *$ metabolic syndrome $($ yes $=1 / \mathrm{no}=0)+0.078 *$ type 2 diabetes $($ yes $=2 /$ no $=0)+0.525 * \log$ 
fasting serum insulin $(\mathrm{mU} / \mathrm{L})+0.521 * \log$ fasting serum AST $(\mathrm{U} / \mathrm{L})-0.454 * \log (\mathrm{AST} / \mathrm{ALT})$ ”. A significant correlation was found between LFC identified by proton magnetic resonance spectroscopy and LFC calculated by the equation $(\mathrm{r}=0.7, \mathrm{P}<0.0001)[22]$.

Table 1: Demographic data of patients and controls at baseline

\begin{tabular}{|l|c|c|c|}
\hline & $\begin{array}{c}\text { Control group } \\
(\mathrm{n}=20)\end{array}$ & $\begin{array}{c}\text { Pioglitazone group } \\
(\mathrm{n}=20)\end{array}$ & $\begin{array}{c}\text { Metformin group } \\
(\mathrm{n}=20)\end{array}$ \\
\hline Sex $(\mathrm{F} / \mathrm{M})$ & $6 / 14$ & $5 / 15$ & $6 / 14$ \\
\hline Age $($ years $)$ & $43.1 \pm 8.1$ & $47.2 \pm 7.9$ & $45.8 \pm 8.3$ \\
\hline BMI $\left(\mathrm{kg} / \mathrm{m}^{2}\right)$ & $22.99 \pm 1.05$ & $33.98 \pm 3.84^{\mathrm{a}}$ & $33.68 \pm 4.49^{\mathrm{a}}$ \\
\hline FBG $(\mathrm{mg} / \mathrm{dl})$ & $91.8 \pm 4.1$ & $95.5 \pm 4.9^{\mathrm{a}}$ & $94.6 \pm 4.0$ \\
\hline
\end{tabular}

Table 1: Data are presented as mean \pm SD. Control group: healthy subjects; Pioglitazone group: patients with NAFLD treated with pioglitazone $45 \mathrm{mg} /$ day; Metformin group: patients with NAFLD treated with metformin 20mg/kg/day. M: male; F: female; BMI: body mass index; FBG: fasting blood glucose. a: Significant versus control at $\mathrm{p}<0.05$.

\section{Statistical Analysis}

Data were analyzed using SPSS statistical package version 22.0, IBM Corporation Software Group, USA. The Kolmogorov-Smirnov test was used to evaluate the normal distribution of the continuous variables. Paired Student's t-test was used to assess any significant difference between each group at baseline and after 6 months of treatment course. One way analysis of variance (ANOVA) test followed by Bonferroni test was used to assess any significant difference among the three groups together. Pearson's correlation test was used to assess the correlation between measured parameters after the effective intervention. Fisher's exact test was used for statistical analysis of the improvement in liver steatosis that evaluated by ultrasound. The results were expressed as mean \pm SD. All $p$ values were two-tailed and $p<0.05$ was considered statistically significant.

\section{RESULTS}

The results are summarized in Table (2). At baseline, there were significant differences between the control group and intervention groups (pioglitazone group and metformin group) in biochemical parameters and LFC. However, there were no significant difference between pioglitazone group and metformin group at baseline and after six months of treatment. Treatment of NAFLD patients for six months resulted in a significant increase in BMI $(\mathrm{p}<0.05)$ in pioglitazone group but a significant decrease in BMI $(\mathrm{p}<0.05)$ in metformin group. The NAFLD patients also exhibited a significant decrease in serum fasting insulin and HOMA-IR (p $<0.05$ ) in pioglitazone group and metformin group versus their pretreatment values (Table 2).

Treatment for six months also resulted in a significant decrease in serum level of liver enzymes; AST (p $<0.01)$ and ALT $(\mathrm{p}<0.01)$ and a significant decrease in fasting plasma FFA and LFC ( $\mathrm{p}<0.01)$ compared to pretreatment values in pioglitazone and metformin groups. There was a significant decrease in serum LDL-C ( $\mathrm{p}$ $<0.05)$ in metformin group, but not in pioglitazone group. Serum levels of HDL-cholesterol and adiponectin were significantly elevated $(\mathrm{p}<0.01)$ in both treatment groups versus the corresponding pretreatment values.

Pearson's correlation analysis regarding post treatment groups revealed a significant positive correlation between BMI and each of HOMA-IR \& LFC\% and between HOMA-IR and each of FFA \&LFC\%. A significant negative correlation was found between adiponectin and each of BMI \&LFC\% (Figure 1).

Table 2: Clinical variables at baseline and after 6 months

\begin{tabular}{|c|c|c|c|c|c|}
\hline Variables & Control group & \multicolumn{2}{|c|}{ Pioglitazone group } & \multicolumn{2}{c|}{ Metformin group } \\
\hline & $(\mathrm{n}=20)$ & \multicolumn{2}{|c|}{$(\mathrm{n}=20)$} & \multicolumn{2}{|c|}{$(\mathrm{n}=20)$} \\
\hline & Baseline & Baseline & 6 months & Baseline & 6 months \\
\hline BMI $\left(\mathrm{kg} / \mathrm{m}^{2}\right)$ & $22.99 \pm 1.05$ & $33.98 \pm 3.84^{\mathrm{a}}$ & $34.59 \pm 4.14^{\mathrm{a}, \mathrm{b}^{*}}$ & $33.68 \pm 4.49^{\mathrm{a}}$ & $32.82 \pm 4.61^{\mathrm{a}, \mathrm{b}^{*}}$ \\
\hline FBG $(\mathrm{mg} / \mathrm{dl})$ & $91.75 \pm 4.07$ & $95.5 \pm 4.87^{\mathrm{a}}$ & $91.75 \pm 3.82^{\mathrm{b}^{* *}}$ & $94.55 \pm 4.02$ & $91.85 \pm 3.85^{\mathrm{b}^{*}}$ \\
\hline Fasting insulin $(\mu \mathrm{IU} / \mathrm{ml})$ & $8.3 \pm 1.10$ & $15.49 \pm 3.14^{\mathrm{a}}$ & $11.94 \pm 2.34^{\mathrm{a} \mathrm{b}^{* *}}$ & $16.09 \pm 4.24^{\mathrm{a}}$ & $12.99 \pm 3.16^{\mathrm{a}, \mathrm{b}^{* *}}$ \\
\hline HOMA-IR & $1.88 \pm 0.29$ & $3.67 \pm 0.87^{\mathrm{a}}$ & $2.71 \pm 0.57^{\mathrm{a} \mathrm{b}^{* *}}$ & $3.76 \pm 1.08^{\mathrm{a}}$ & $2.96 \pm 0.79^{\mathrm{a}, \mathrm{b}^{* *}}$ \\
\hline AST $(\mathrm{U} / \mathrm{L})$ & $30.9 \pm 4.0$ & $48.7 \pm 15.76^{\mathrm{a}}$ & $34.8 \pm 9.3^{\mathrm{b}^{* *}}$ & $48.05 \pm 14.05^{\mathrm{a}}$ & $36.3 \pm 8.28^{\mathrm{b}^{* *}}$ \\
\hline ALT $(\mathrm{U} / \mathrm{L})$ & $38.9 \pm 1.9$ & $57.8 \pm 15.04^{\mathrm{a}}$ & $38.35 \pm 10.7^{\mathrm{b}^{* *}}$ & $59.1 \pm 15.99^{\mathrm{a}}$ & $42.5 \pm 7.72^{\mathrm{b}^{* *}}$ \\
\hline TC $(\mathrm{mg} / \mathrm{dl})$ & $173.4 \pm 7.4$ & $201.8 \pm 27.72^{\mathrm{a}}$ & $193.95 \pm 27.62^{\mathrm{a}}$ & $200.05 \pm 28.68^{\mathrm{a}}$ & $190.6 \pm 22.76^{\mathrm{a}}$ \\
\hline LDL-C $(\mathrm{mg} / \mathrm{dl})$ & $100.5 \pm 8.3$ & $126.3 \pm 27.35^{\mathrm{a}}$ & $113.5 \pm 28.44$ & $123.6 \pm 31.44^{\mathrm{a}}$ & $108.45 \pm 24.07^{\mathrm{b}^{*}}$ \\
\hline
\end{tabular}


Pioglitazone versus metformin in Egyptian non diabetic patients

\begin{tabular}{|c|c|c|c|c|c|}
\hline HDL-C $(\mathrm{mg} / \mathrm{dl})$ & $52.2 \pm 4.2$ & $44.4 \pm 7.3^{\mathrm{a}}$ & $51.35 \pm 8.95^{\mathrm{b}^{* *}}$ & $41.50 \pm 6.55^{\mathrm{a}}$ & $48.05 \pm 6.28^{\mathrm{b}^{* *}}$ \\
\hline TGs $(\mathrm{mg} / \mathrm{dl})$ & $103.8 \pm 21.9$ & $155.3 \pm 46.7^{\mathrm{a}}$ & $145.5 \pm 43.1^{\mathrm{a}}$ & $174.8 \pm 47.3^{\mathrm{a}}$ & $170.5 \pm 39.6^{\mathrm{a}}$ \\
\hline Adiponectin $(\mu \mathrm{g} / \mathrm{ml})$ & $10.9 \pm 0.7$ & $6.86 \pm 1.83^{\mathrm{a}}$ & $8.04 \pm 2.15^{\mathrm{a}, \mathrm{b}^{* *}}$ & $7.26 \pm 2.1^{\mathrm{a}}$ & $8.21 \pm 2.04^{\mathrm{a}, \mathrm{b}^{* *}}$ \\
\hline Fasting plasma FFA $(\mathrm{ng} / \mathrm{ml})$ & $258.4 \pm 21.9$ & $476.1 \pm 125.7^{\mathrm{a}}$ & $342.6 \pm 67.9^{\mathrm{a}, \mathrm{b}^{* *}}$ & $479.6 \pm 143.5^{\mathrm{a}}$ & $372.5 \pm 87.2^{\mathrm{a}, \mathrm{b}^{* *}}$ \\
\hline Liver fat content $\%$ & $4.96 \pm 0.38$ & $8.17 \pm 1.77^{\mathrm{a}}$ & $5.99 \pm 1.37^{\mathrm{a}, \mathrm{b}^{* *}}$ & $8.14 \pm 1.75^{\mathrm{a}}$ & $6.41 \pm 1.16^{\mathrm{a}, \mathrm{b}^{* *}}$ \\
\hline
\end{tabular}

Table 2: Data are presented as mean \pm SD. Control group: healthy subjects; Pioglitazone group: patients with NAFLD treated with pioglitazone $45 \mathrm{mg}$ /day; Metformin group: patients with NAFLD treated with metformin 20mg/kg/day. BMI: Body mass index; FBG: Fasting blood glucose; TC: Total cholesterol; TGs: Triglycerides; LDL-C: Low-density lipoprotein cholesterol; HDL-C: High-density lipoprotein cholesterol; HOMA-IR: Homeostatic model of assessment of insulin resistance; FFA: Free fatty acids; ALT: Alanine aminotransferase; AST: Aspartate aminotransferase. a: Significant versus control at $\mathrm{p}<0.05$; b: Significant versus pretreatment. $* \mathrm{P}<0.05, * * \mathrm{P}<0.01$ compared with corresponding baseline values (paired sample t-test).
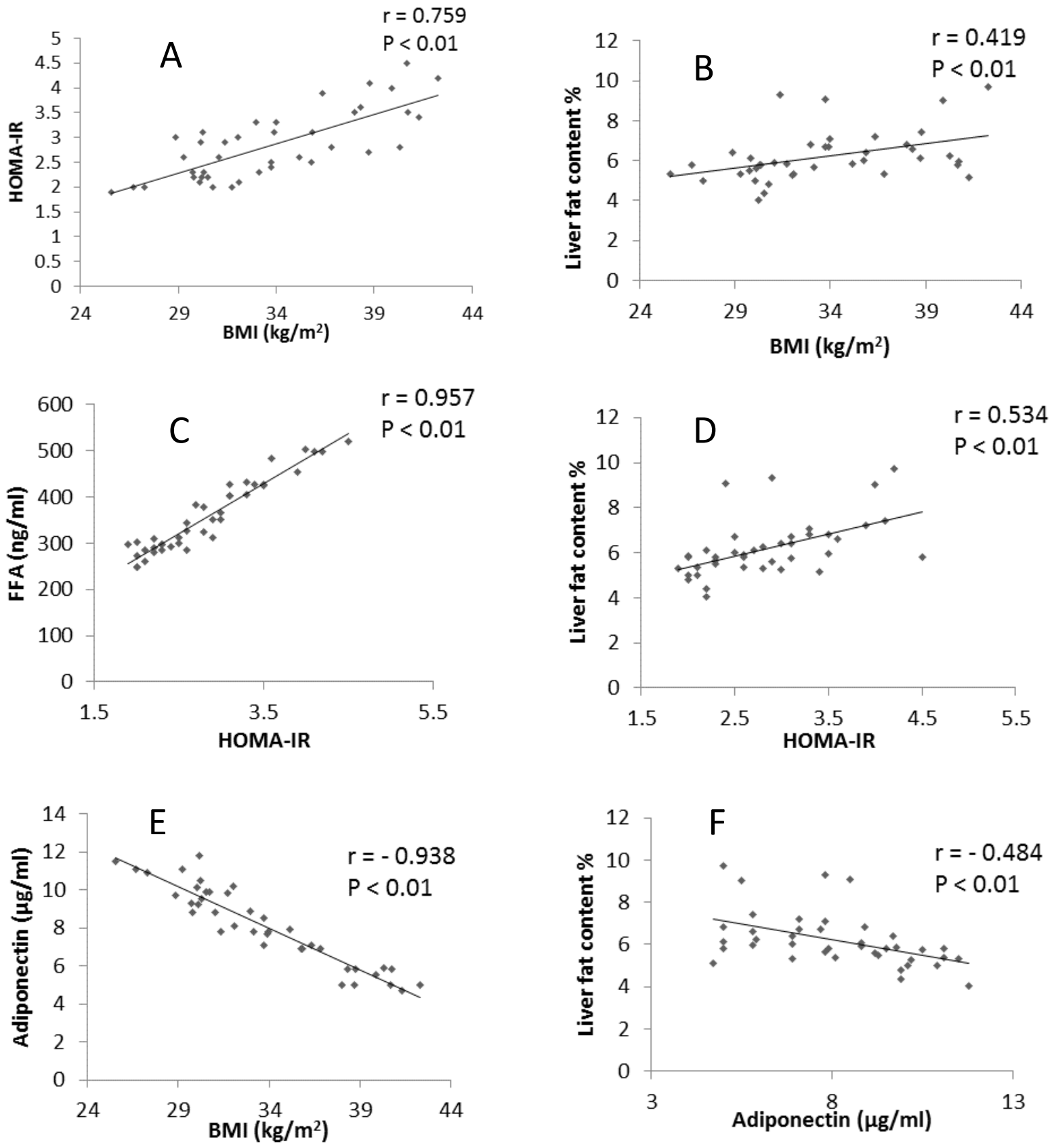
Figure (1): Correlation between measured parameters. (A) Positive correlation between BMI and HOMA-IR (B) Positive correlation between BMI and LFC\%. (C) Positive correlation between HOMA-IR and FFA. (D) Positive correlation between HOMA-IR and LFC\%. (E) Negative correlation between adiponectin and BMI. (F) Negative correlation between adiponectin and LFC\%.

\section{DISCUSSION}

Insulin resistance may contribute to the development of fatty liver by impairing the ability of insulin to suppress lipolysis, leading to increased delivery of FFAs to the liver [5]. In the present study, we assessed whether the insulin sensitizers pioglitazone and metformin would affect white adipose tissue metabolism and liver fat content in overweight/obese non diabetic patients with NAFLD.

The NAFLD patients included in the present study showed increased value of BMI and elevated insulin level with HOMA-IR >2.5 indicating insulin resistance. Treatment of NAFLD with metformin or pioglitazone, herein, showed a significant decrease in fasting insulin and HOMA-IR. These results could be explained by the reported mechanism of action of metformin and pioglitazone, which both considered insulin sensitizers. Our data were in accordance with previous reports. Razavizade et al. [7] revealed that four-months of treatment of NAFLD patients with metformin (1g/day) or pioglitazone $(30 \mathrm{mg} /$ day) led to a significant decrease in HOMAIR in both groups. Bugianesi et al. [23] reported that metformin ( $2 \mathrm{~g} /$ day) was effective in decreasing insulin level in fifty-five NAFLD patients for twelve-months. Patients with NAFLD and impaired glucose tolerance or type II diabetes mellitus were included in the study of Belfort et al. [24], who demonstrated that treatment with pioglitazone $(45 \mathrm{mg} / \mathrm{d})$ was better than placebo group in reducing fasting plasma insulin level after 6 months. Another study by Garinis et al. [25] revealed that six-month treatment of NAFLD patients with metformin $(1 \mathrm{~g} / \mathrm{d})$ plus diet was effective in improving HOMA-IR, with no effect on insulin level.

Although the plasma FFA were significantly higher in NAFLD patients than the control value, the decrease of HOMA-IR in the present study by six-months treatment with either metformin or pioglitazone was accompanied by a significant decrease in plasma FFA in both treated groups. Pearson's correlation analysis supported our data and indicated a significant positive correlation between FFA and HOMA-IR in post treatment groups. These results were in agreement with those achieved by Belfort et al. [24], who found a significant reduction in fasting plasma FFA level by $17 \%$ in the group treated with pioglitazone $(45 \mathrm{mg} /$ day) compared to placebo in patients with NAFLD. Promrat et al. [26] also found a significant reduction in FFA level by $16 \%$ compared to baseline in eighteen pioglitazone-treated patients $(30 \mathrm{mg} / \mathrm{day})$ with NAFLD for forty-eight weeks. Our results were comparable and showed a decrease of FFA by $28 \%$ in pioglitazone group and $22 \%$ in metformin group.

The reduction of plasma FFA by pioglitazone could be explained by that pioglitazone binds to PPAR- $\gamma$ and stimulates peripheral adipocytes to increase their uptake of free fatty acids, which leads to reduction in the fat stored in muscle, liver and visceral fat deposits [27]. Pioglitazone attenuates liver fibrosis by suppressing the activation of hepatic stellate cells into activated myofibroblasts suggesting an additional direct hepatoprotective effect [27]. Pioglitazone also decreased serum tumor necrosis factor alpha (TNF- $\alpha$ ), thereby it could prevent inflammation and necrosis which have been implicated in the pathophysiology of NASH [9].

Metformin has antilipolytic effect, reducing plasma FFA concentration [25]. Previous study showed a significant reduction of fasting plasma FFA in nineteen patients with insulin resistance by $850 \mathrm{mg}$ (b.i.d) of metformin for six weeks [28]. This can be explained by the effect of metformin through the activation of liver kinase B1, leading to up regulation of adenosine monophosphate-activated protein kinase (AMPK) [21]. Activation of AMPK by metformin, not only inhibits gluconeogenesis, but also inhibits the sterol regulatory element-binding protein-1c (SREBP-1c), which is a transcription factor for genes involved in fatty acid synthesis and is inappropriately increased in liver of NAFLD patients [29]. AMPK also inactivates acetyl-CoA carboxylase and HMG CoA reductase reducing fatty acid and cholesterol synthesis capabilities [21].

The obtained biochemical results in our study regarding reduction of plasma FFA and improvement of insulin sensitivity were further strenghthed and confirmed by liver ultrasound. Liver steatosis, as evaluated by ultrasound, herein, improved in 13 of $20(65 \%)$ patients of the pioglitazone group ( $\mathrm{P}<0.0001$, final versus baseline) and in 5 of 20 (25\%) patients of the metformin group ( $\mathrm{P}<0.05$, final versus baseline). Meanwhile, the present results showed that pioglitazone was superior to metformin in improving liver stestosis. Garinis et al. [25] showed that liver hyperechogenicity (improved /disappeared) in ultrasound in NAFLD patients treated with metformin $(1 \mathrm{~g} / \mathrm{d})$ plus diet compared with diet alone for six months. Previous studies of liver biopsy samples provided supporting data and indicated improvement in liver histological features after two years treatment of NAFLD patients with pioglitazone (30mg/day) [30] and twelve months treatment with metformin (2g/day) [31].

The amelioration of liver steatosis in the present study was also reflected by the significant reduction in serum level of ALT and AST indicating improvement of liver function in both treatment groups. These results were in accordance with those of Aithal et al. [32], who showed that there was a significant reduction in ALT level after pioglitazone (30 mg/day) treatment of NAFLD patients for one year. Al-Gharabally et al. [33] 
compared the effects of pioglitazone (15-45 mg/day) versus weight reduction diet for forty-eight months on nineteen NAFLD patients and found that AST and ALT levels decreased in the pioglitazone group but not in diet group. Lutchman et al. [34] demonstrated that the mean serum AST and ALT levels significantly reincreased after forty-eight weeks of discontinuation of pioglitazone although they significantly decreased after forty-eight weeks of treatment with pioglitazone (30 mg /day) in twenty-one NAFLD patients. The decrease of serum AST and ALT levels was also reported in NAFLD patients treated with metformin (1.7 g/d) for sixmonths [35] and in NAFLD patients treated with metformin $(2 \mathrm{~g} / \mathrm{d})$ for twelve-months [23].

The beneficial effect of pioglitazone and metformin in reducing liver steatosis was further confirmed by the observed significant reduction of liver fat content in the treated NAFLD patients as shown by our data. These results were in accordance with Razavizade et al. [7], who revealed a significant decrease in liver fat content in NAFLD patients treated with either metformin or pioglitazone. The study of Belfort et al. [24] measured liver fat content by magnetic resonance spectroscopy, which showed a significant reduction by $54 \%$ in pioglitazone treated NAFLD patients.

Considering the lipid profile, the present results indicated that both pioglitazone and metformin groups showed insignificant change in serum triglyceride and cholesterol after six months treatment. These findings were in accordance with the results of previous trials that illustrated that serum TG level did not change significantly following pioglitazone treatment [7] or metformin treatment [25] in NAFLD patients. As observed by our data, the post treatment level of LDL-C decreased significantly in metformin group but not in pioglitazone group. These results were consistent with the study of Haukeland et al. [36] on forty-eight NAFLD patients where a significant decrease in serum LDL-C was observed in group treated with metformin (2.5-3 $\mathrm{g}$ /day) versus placebo group. On the other hand, the current data indicated that serum HDL-C increased significantly in both metformin and pioglitazone-treated NAFLD groups.

Some adipose tissue-released cytokines are implicated in the development of insulin resistance and increased rate of lipogenesis. Adiponectin is an adipocyte-derived plasma protein reported to improve muscular and hepatic insulin sensitivity [37]. Adiponectin level is reduced in obese patients, and T2DM, and its plasma concentration is inversely related to body weight, especially to visceral adiposity [37]. The present data were in line and demonstrated that a significant negative correlation was obtained between BMI and adiponectin in NAFLD patients. NAFLD patients involved in our study exhibited a significantly reduced serum level of adiponectin at the start of study versus normal controls, which was associated with insulin resistance in the selected patients. In liver, adiponectin increases FFA oxidation, and decreases de novo lipogenesis through the activation of AMP activated protein kinase [38]. So, low level of adiponectin may play a role in the pathogenesis of NAFLD by decreasing the oxidation of fatty acids in the liver. Adiponectin levels have been found to be significantly lower in subjects with NAFLD or NASH compared with controls [38].

The obtained results, herein, showed a significant increase in serum level of adiponectin in both treatment groups and a significant negative correlation between liver fat content and adiponectin level. This is consistent with Bugianesi et al. [39] and Bajaj et al. [40]. Adiponectin exerts anti-inflammatory and anti-fibrotic action. Its anti-inflammatory action is mainly achieved by suppressing pro-inflammatory cytokines (e.g., TNFaand IL-6) which have a role in the development of liver injury in patients with NASH, and its anti-fibrotic action is mainly achieved via reducing hepatic stellate cells activation [38].

Regarding BMI, the current data showed a significant increase in BMI in the pioglitazone group. Promrat et al. [26] study supported this view and documented that most NAFLD patients had a decrease in liver fat, but an increase in total body adiposity with a significant increase in BMI after treatment with pioglitazone. Weight gain is considered as a common side effect of pioglitazone as thiazolidinediones are agonists for PPAR- $\gamma$ receptor that expressed in adipocytes at the highest level. So, they make redistribution of lipid from muscle and liver to peripheral adipocytes [41]. On the contrary, treatment of NAFLD patients with metformin in the current work resulted in a significant decrease in BMI. These results may be contributed by improving insulin sensitivity with metformin. Seifarth et al. [42] found that treatment of obese insulin resistant non diabetic patients with metformin (2.5 g/day) for six months decreased body weight and BMI significantly.

It is worthwhile to report that none of the NAFLD patients included in our study complained of hypoglycemia during the study duration, even though, some of the patients complained of bloating and abdominal pain within the first month without cessation of the drug.

\section{CONCLUSION}

Besides acting as insulin sensitizers, pioglitazone and metformin exhibited a hepatoprotective effect in nondiabetic NAFLD patients. Both drugs decreased liver fat content, modified lipid metabolism and managed liver transaminases with no evident hypoglycemia. So, they could be utilized as a treatment option to guard against fat deposition in the liver or progression of steatosis to fibrosis and cirrhosis. However, further studies are required to examine the molecular mechanisms underlying the beneficial effect of the insulin sensitizers in NAFLD patients. 
Statement of conflict of interest: Nothing to declare.

\section{REFERENCES}

[1] Ahmed M. Non-alcoholic fatty liver disease in 2015. World J Hepatol 2015; 7: 1450-1459

[2] Hashimoto E, Tokushige K, Ludwig J. Diagnosis and classification of non-alcoholic fatty liver disease and non-alcoholic steatohepatitis: Current concepts and remaining challenges. Hepatol Res 2015; 45: 2028

[3] Streba LAM, Vere CC, Rogoveanu I et al. Nonalcoholic fatty liver disease, metabolic risk factors, and hepatocellular carcinoma: An open question. World J Gastroenterol 2015; 21: 4103-4110

[4] Takahashi Y, Sugimoto K, Inui $\mathrm{H}$ et al. Current pharmacological therapies for nonalcoholic fatty liver disease/ nonalcoholic steatohepatitis. World J Gastroenterol 2015; 21: 3777-3785

[5] Schwenger KJP, Allard JP. Clinical approaches to non alcoholic fatty liver disease. World J Gastroenterol 2014; 20: 1712-1723

[6] Ozturk ZA, Kadayifci A. Insulin sensitizers for the treatment of non-alcoholic fatty liver disease. World J Hepatol 2014; 6: 199-206

[7] Razavizade M, Jamali R, Arj A et al. The effect of pioglitazone and metformin on liver function tests, insulin resistance, and liver fat content in nonalcoholic fatty liver disease: a randomized double blinded clinical trial. Hepat Mon 2013; 13: e9270

[8] Fruci B, Giuliano S, Mazza A et al. Nonalcoholic fatty liver: a possible new target for type 2 diabetes prevention and treatment. Int J Mol Sci 2013; 14: 22933-22966

[9] Le T-A, Loomba R. Management of non-alcoholic fatty liver disease and steatohepatitis. J Clin Exp Hepatol 2012; 2: 156-173

[10] Trinder P. Determination of glucose in blood using glucose oxidase with an alternative oxygen acceptor. Ann clin Bio chem 1969; 6: 24-25

[11] Reitman A, Frankel S. Guidelines on standard operating Procedures of transaminases. Am J Clin Pathol 1957; 28: 56-63

[12] Tsao TS, Lodish HF, Fruebis J. ACRP30, a new hormone controlling fat and glucose metabolism. Eur J Pharmacol 2002; 440: 213-221

[13] Andersen L, Dinesen B, Jørgensen PN et al. Enzyme immunoassay for intact human insulin in serum or plasma. Clin Chem 1993; 39: 578-582

[14] Watson D. A simple method for the determination of serum cholesterol. Clin Chim Acta 1960; 5: 637643

[15] Fossati P, Prencipe L. Serum triglycerides determined colorimetrically with an enzyme that produces hydrogen peroxide. Clin Chem 1982; 28: 2077-2080

[16] Warnick GR, Wood PD. National Cholesterol Education Program recommendations for measurement of high-density lipoprotein cholesterol: executive summary. The National Cholesterol Education Program Working Group on Lipoprotein Measurement. Clin Chem 1995; 41: 1427-1433

[17] Friedewald WT, Levy RI, Fredrickson DS. Estimation of the concentration of low-density lipoprotein cholesterol in plasma, without use of the preparative ultracentrifuge. Clin Chem 1972; 18: $499-502$

[18] Ray S, Talukdar A, Sonthalia N et al. Serum lipoprotein ratios as markers of insulin resistance: A study among non-diabetic acute coronary syndrome patients with impaired fasting glucose. Indian J Med Res 2015; 141: 62-67

[19] Palmentieri B, De Sio I, La Mura V et al. The role of bright liver echo pattern on ultrasound B-mode examination in the diagnosis of liver steatosis. Dig Liver Dis 2006; 38: 485-489

[20] Roldan-Valadez E, Favila R, Martinez-Lopez M et al. Imaging techniques for assessing hepatic fat content in nonalcoholic fatty liver disease. Ann Hepatol 2008; 7: 212-220

[21] Mazza A, Fruci B, Garinis GA et al. The role of metformin in the management of NAFLD. Exp Diabetes Res 2012; 2012:716404

[22] Kotronen A, Peltonen M, Hakkarainen A et al. Prediction of non-alcoholic fatty liver disease and liver fat using metabolic and genetic factors. Gastroenterology 2009; 137: 865-872

[23] Bugianesi E, Gentilcore E, Manini R et al. A randomized controlled trial of metformin versus vitamin E or prescriptive diet in nonalcoholic fatty liver disease. Am J Gastroenterol 2005; 100: 1082-1090

[24] Belfort R, Harrison SA, Brown K et al. A placebo-controlled trial of pioglitazone in subjects with nonalcoholic steatohepatitis. N Engl J Med 2006; 355: 2297-2307

[25] Garinis GA, Fruci B, Mazza A et al. Metformin versus dietary treatment in nonalcoholic hepatic steatosis: a randomized study. Int J Obes (Lond) 2010; 34: 1255-1264

[26] Promrat K, Lutchman G, Uwaifo GI et al. A pilot study of pioglitazone treatment for nonalcoholic steatohepatitis. Hepatology 2004; 39: 188-196 
[27] Hardy T, Anstee QM, Day CP. Nonalcoholic fatty liver disease: new treatments. Curr Opin Gastroenterol 2015; 31: 175-183

[28] Castro Cabezas M, van Wijk JP, Elte JW et al. Effects of metformin on the regulation of free fatty acids in insulin resistance: a double-blind, placebo-controlled study. J Nutr Metab 2012; 2012:394623

[29] Phielix E, Szendroedi J, Roden M. The role of metformin and thiazolidinediones in the regulation of hepatic glucose metabolism and its clinical impact. Trends Pharmacol Sci 2011; 32: 607-616

[30] Sanyal AJ, Chalasani N, Kowdley KV et al. Pioglitazone, Vitamin E, or Placebo for Nonalcoholic Steatohepatitis. N Engl J Med 2010; 362: 1675-1685

[31] Loomba R, Lutchman G, Kleiner DE et al. Clinical trial: pilot study of metformin for the treatment of non-alcoholic steatohepatitis. Aliment Pharmacol Ther 2009; 29: 172-182

[32] Aithal GP, Thomas JA, Kaye PV et al. Randomized, placebo-controlled trial of pioglitazone in nondiabetic subjects with nonalcoholic steatohepatitis. Gastroenterology 2008; 135: 1176-1184

[33] Al-Gharabally A, O'Brien CB, Acosta RC. A pilot study of pioglitazone for the treatment of nonalcoholic fatty liver disease. Hepat Mon 2007; 7: 131-137

[34] Lutchman G, Modi A, Kleiner DE et al. The effects of discontinuing pioglitazone in patients with nonalcoholic steatohepatitis. Hepatology 2007; 46: 424-429

[35] Uygun A, Kadayifci A, Isik $\mathrm{T}$ et al. Metformin in the treatment of patients with non-alcoholic Steatohepatitis. Aliment Pharmacol Ther 2004; 19: 537-544

[36] Haukeland JW, Konopski Z, Eggesbø HB et al. Metformin in patient with non-alcoholic fatty liver disease: a randomized, controlled trial. Scand J Gastroenterol 2009; 44: 853-860

[37] Abenavoli L, Di Renzo L, Guzzi PH et al. Non-alcoholic fatty liver disease severity, central fat mass and adinopectin: a close relationship. Clujul Med 2015; 88: 489-493

[38] Polyzos SA, Kountouras J, Mantzoros CS. Adipokines in nonalcoholic fatty liver disease. Metabolism 2015. pii: S0026-0495(15)00351-0.

[39] Bugianesi E, Pagotto U, Manini R et al. Plasma adiponectin in nonalcoholic fatty liver is related to hepatic insulin resistance and hepatic fat content, not to liver disease severity. J Clin Endocrinol Metab 2005; 90: 3498-3504

[40] Bajaj M, Suraamornkul S, Piper P et al. Decreased plasma adiponectin concentrations are closely related to hepatic fat content and hepatic insulin resistance in pioglitazone-treated type 2 diabetic patients. J Clin Endocrinol Metab 2004; 89: 200-206

[41] Van Wagner LB, Rinella ME. The role of insulin-sensitizing agents in the treatment of nonalcoholic steatohepatitis. Therap Adv Gastroenterol 2011; 4: 249-263

[42] Seifarth C, Schehler B, Schneider HJ. Effectiveness of metformin on weight loss in non-diabetic individuals with obesity. Exp Clin Endocrinol. Diabetes 2013; 121: 27-31 